\title{
A New FE-Model for the Investigation of Bond Formation and Failure in Roll Bonding Processes
}

\author{
Michael Pietryga ${ }^{1, a^{*}}$, Johannes Lohmar ${ }^{1, b}$ and Gerhard Hirt ${ }^{1, c}$ \\ ${ }^{1}$ Institute of Metal Forming (IBF), RWTH Aachen University, Aachen, Germany \\ apietryga@ibf.rwth-aachen.de, 'bohmar@ibf.rwth-aachen.de, 'chirt@ibf.rwth-aachen.de
}

Keywords: Roll bonding, Aluminum, material modelling, Abaqus, UINTER

\begin{abstract}
Roll bonding is a process to join two or more different materials permanently in a rolling process. A typical industrial application is the manufacturing of aluminum sheets for heat exchangers in cars where the solder is joined onto a base layer by roll bonding. From a modelling point of view the challenge is to describe the bond formation and failure of the different material layers within a FE-process model. Most methods established today either tie the different layers together or treat them as completely separate. The problem for both assumptions is that they are not applicable to describe the influence of tangential stresses that can cause layer shifting and occur in addition to the normal stresses within the roll gap. To overcome these restrictions in this paper a 2D FE-model is presented that integrates an adapted contact formulation being able to join two bodies that are completely separated at the start of the simulation. The contact formulation is contained in a user subroutine that models bond formation by adhesion in dependence of material flow and load. Additionally if the deformation conditions are detrimental already established bonds can fail. This FE-model is then used to investigate the process boundaries of the first passes of a typical rolling schedule in terms of achievable height reductions. The results show that passes with unfavorable height reduction introduce tensile and shear stresses that can lead to incomplete bonding or can even destroy the bond entirely. It is expected that, with adequate calibration, the developed FEmodel can be used to identify conditions that are profitable for bond formation in roll bonding prior to production and hence can lead to shorter rolling schedules with higher robustness.
\end{abstract}

\section{Introduction}

In roll bonding two or more separate metal strips or plates are fused together via the plastic deformation generated in the roll gap of a rolling mill. Typically the top-layers of such a package are thinner than the base-layer. The formation of a firm bond is dependent on a plentitude of influencing factors most of which can be set by the roll stand setup. To optimize the roll bonding process it is hence crucial to model the influence of the acting deformation and stresses on bonding and de-bonding. Tangential stresses can cause relative motion between the bodies in the roll gap and eventually lead to failure. Thus, modelling needs to account for relative motion which rules out elementary approaches like the slab method. This paper proposes a FE-model using a user subroutine to model bond formation and failure at the interface of the bodies based on parameters obtained from laboratory experiments. The capabilities of this model are demonstrated based on variations of height reductions and top-layer thickness. By adopting the model for multiple roll passes in the future, it will be possible to determine optimal process parameters for roll bonding via simulation rather than via time and cost intensive trial and error.

\section{State of the Art}

Besides the influencing factors set by the roll stand setup i.e. roll gap geometry, pass reduction and thickness ratio there are additional influencing factors for roll bonding such as the type of metals to be joined together, the initial temperature, the surface state, the elastic and plastic material properties, the microstructure, the heat expansion coefficients and friction. Hence, it is very difficult to describe the roll bonding process. In the past analytical models that only consider a fraction of these factors and solely predict integral values like the roll force were proposed by several authors 
[1-4]. None of these models considers the actual bond formation as all models assume the sheets to be bonded at the roll gap exit. A detailed survey of models and processes for roll bonding was also delivered by Schmidtchen et. al [5]. Bay et. al [6] were the first to suggest a comprehensive model that tackled the bond formation in the roll gap, however de-bonding and failure is not considered. Hence, a model formulation is needed that accounts for stress and time dependent adhesion and decohesion in the context of a FE-based process description. Such a model inspired by the work of Bay et. al [6] and based on the Abaqus used subroutine UINTER was proposed by Bambach et. al [7]. An enhanced version is presented in this paper.

\section{FE-model for Bonding and Failure in Rolling Processes}

In this paper the FE-tool ABAQUS is used to model the symmetrical roll bonding of three separate aluminum layers. Although ABAQUS built-in methods for surface-based bonding behavior, damage initiation and damage evolution are available, we need a more flexible approach to simulate the evolution of bond strength as a function of the significant variables detailed above. Therefore, the user subroutine UINTER is used here to define mechanical interactions between surfaces. Using this routine, the entire constitutive contact behavior has to be defined by the user. This means, that normal mechanical contact as well as tangential interaction in terms of a friction law have to be implemented first. On top of that this work extends the UINTER definition by a bonding and failure criterion that respects normal stress but also the shear stress acting within the roll gap. In this section the two main parts of the FE-model are described. First, the process model for rolling is shown. Second, the UINTER subroutine to describe bonding and failure at the interface is illustrated in detail.
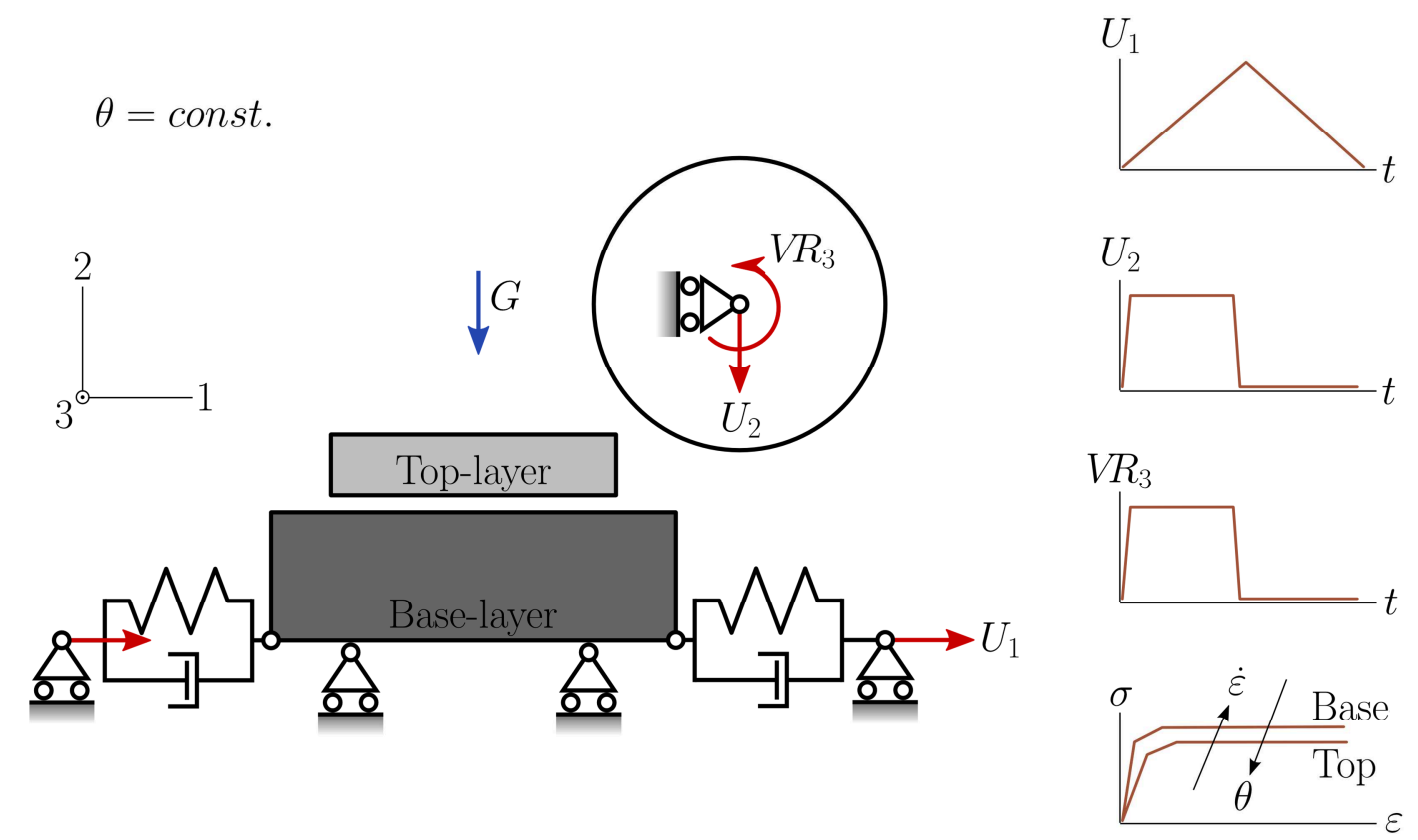

Figure 1: Finite element boundary conditions of the used roll bonding model. Here isothermal condition $\left(\theta=450^{\circ} \mathrm{C}\right)$ is assumed. $U$ denotes displacement and VR rotational velocity.

Figure 1 sketches the 2D process model for a rolling process suitable for symmetrical roll bonding of three layers of aluminum. The entire model consists of three parts. The deformable lower part displays a base-layer which has the higher yield strength compared to the top-layer. The roller consists of analytical rigid surfaces. An implicit dynamic procedure is used for analysis, as this enables gravitational forces to fix top-layer and base-layer onto each other.

Both for the base- and top- layers a strain rate and temperature dependent elasto-plastic material model is used to describe the stress-strain response of the materials. As contact formulation between the two deformable parts a node to surface approach is defined and the user subroutine UINTER 
detailed later is set as contact property. Additively as engineering features spring-dashpot-pairs are included into the entire model on both sides of the lower part in order to control the movement of the entire compound with displacement degrees of freedom and simultaneously minimizing restrictive forces on the model as good as possible.

Figure 2 schematically shows a snapshot of a roll gap in the simulation of roll bonding of two initially separated metal slabs. On the left side a cutaway of a FE-model is visible. It consists of two parts meshed with two different element sizes as required for node-to-surface contact. The top-layer is defined as 'slave' and the base-layer as 'master'-surface. The contour plot illustrates the Mises stress around the roll gap in both layers. In both figures (left and right) the red dashed line emphasizes a segment (A-D) of the contact surfaces of both layers. As indicated with 'Rolling direction' the layer package moves from left to right due to the rotational velocity of the roller and friction between roller and top-layer. Thus point A enters the roll gap first and point D last.

The graph on the right side shows the contact stresses (pressure and shear) along the selected segment A-D. The presented snapshot shows a scenario where the highest normal and tangential stresses arise near point C. Higher normal pressure leads to great plastic deformation and hence significant surface enlargement. Around point B undesired tension stresses (negative contact pressure) in combination with shear stresses can be detected. Hence the graph clearly shows that all stress components need to be considered for a successful modelling of roll bonding.
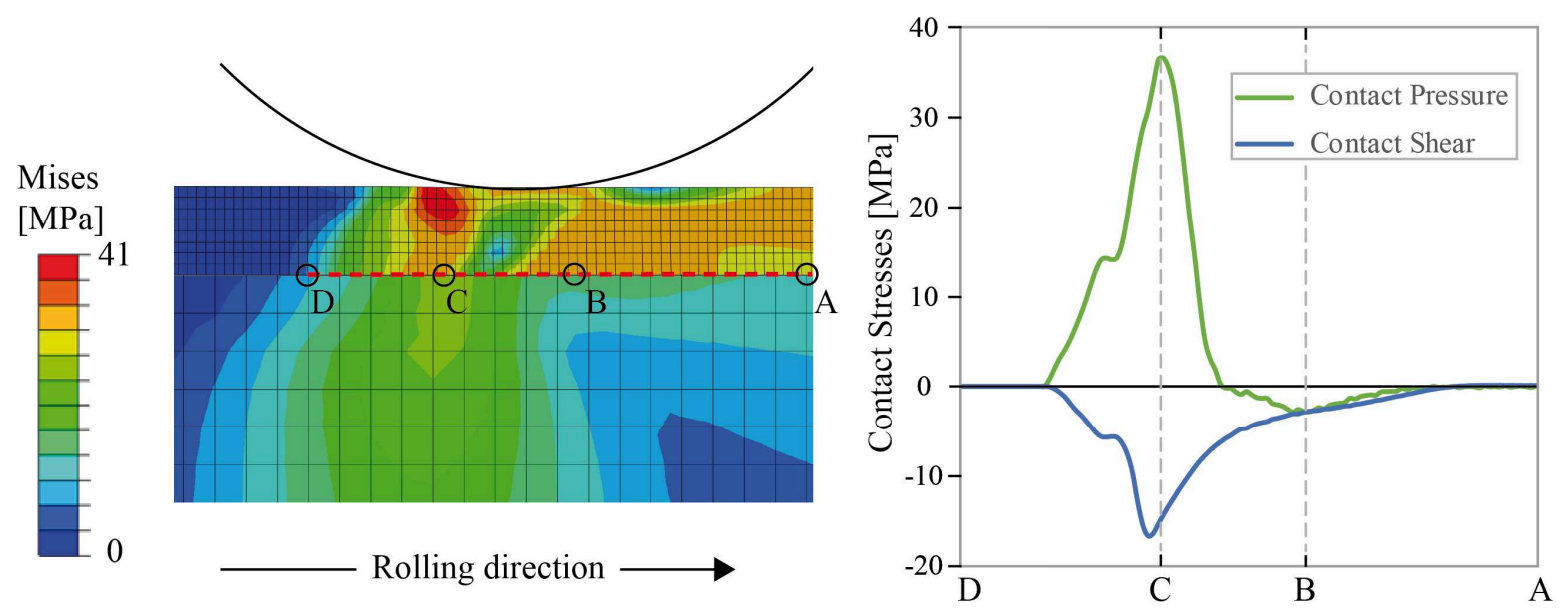

Figure 2: Left: cutaway of the FE-model. Right: Contact pressure and contact shear along the selected segment A-D

Bay et. al suggested that surface enlargement $\varepsilon_{\mathrm{SE}}$ is the most important factor influencing bond strength as it represents the breakup of oxide layers. To obtain the bond strength of an arbitrary material as a function of surface enlargement and temperature for different stress component combinations, complex laboratory experiments detailed elsewhere [8] are necessary. Curves that schematically show the maximum bond strength reached in experiments with two different surface enlargements in the normal-tangential-stress-space relevant for rolling are shown as red lines in Figure 3. Comparing the solid and dotted lines the strong correlation between surface enlargement and resulting maximum bond strength is visible. For tensile stress $(p<0)$, a linear dependence between the nominal bond strength $\sigma_{\mathrm{B}}$ and the tangential bond strength $\tau_{\mathrm{B}}$ can be derived from experimental work. In the compression area $(p>0) \tau_{B}$ can be considered constant. Additively the maximal yield stresses in normal $\left(\sigma_{y, \max }\right)$ and tangential $\left(\tau_{\max }\right)$ direction of top- (green) and baselayer (blue) are illustrated. They show the proportion between the bond strength and yield stress arising for the shown surface enlargements.

Within the process model all mechanical interactions that occur at the interface between the layers are handled by the UNITER subroutine. Surface enlargement can be calculated within UINTER by tracing the displacements of the nodes at the interface. Bond strength in the roll gap will evolve in accordance with the current surface enlargement and the acting stresses calculated for 
each node individually. Lastly frictional behavior between the two aluminum layers is assumed with a coefficient $\mu=0.5$ whereas the friction coefficient between the roller and top-layer is about $\mu=0.2$.

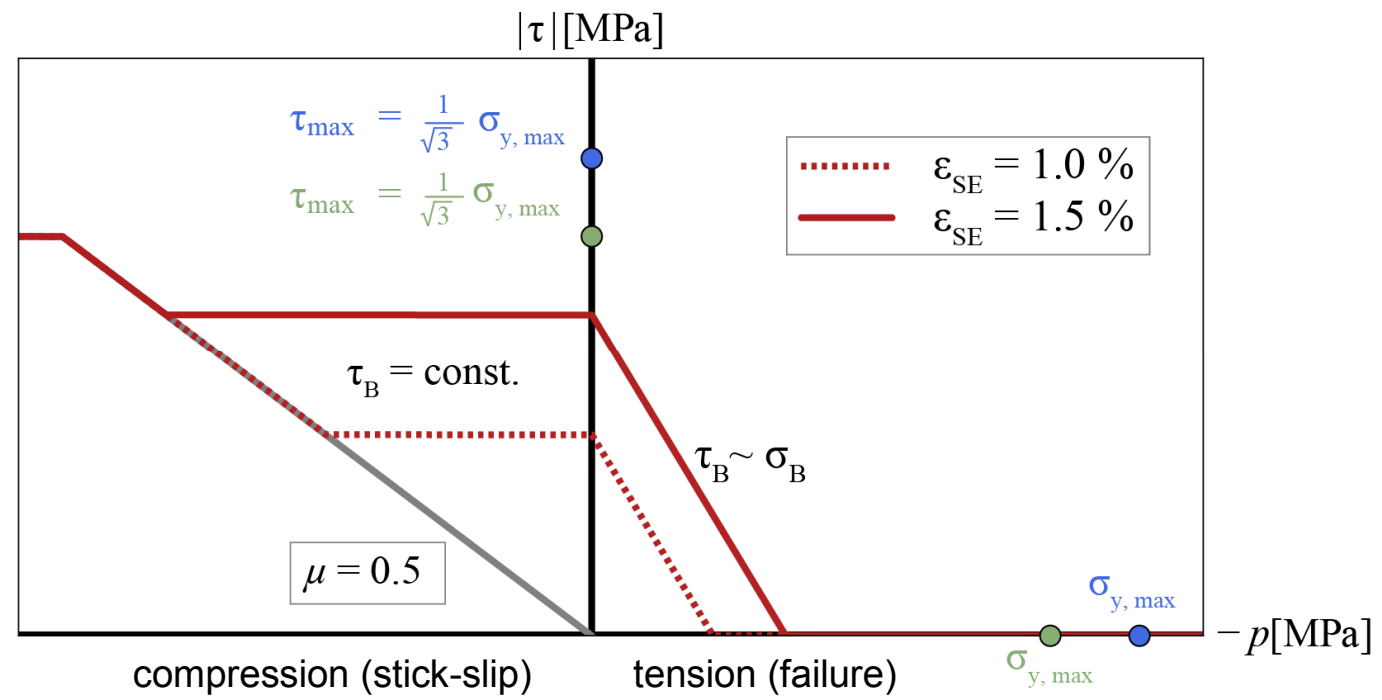

Figure 3: Bond formation and failure based on surface enlargement $\varepsilon_{\mathrm{SE}}$ in the normal-tangential stress space. Frictional behavior between the two layers is assumed with a coefficient $\mu=0.5$. Additionally the maximal yield stresses of both layers are shown.

Wherever the stresses acting in the roll gap exceed the current bond strength failure or slip will occur, thus the red lines in Figure 3 represent boundary states. Due to the coupling to the current surface enlargement the boundary is process dependent and develops individual for each node on the interface. Depending on the normal pressure $p$ different scenarios may arise. For a positive contact pressure $(p>0)$ shear stress may occur until the latter of the friction or failure boundary is reached. In both cases stick-slip due to the Coulomb-Orowan friction model is assumed and the layers remain bonded. For a positive tensile stress $(p<0)$ shear stress is possible until the failure boundary is reached, which due to the acting tension is followed by de-bonding and separation on the layers.

\section{Exemplary Study of Process Parameters}

The main goal of the simulation of the roll bonding process is to optimize the rolling schedule in terms of achievable height reductions. The optimized forming strategy should yield the best bonding strength between both parts possible. Additionally less forming passes are preferable due to energy and temporally costs. The following simulations demonstrate the influence of the typical process parameters height reduction and layer thickness ratio during a single forming pass on the bonding behavior.

Figure 4 shows the bond strength $\sigma_{\mathrm{B}}$ along the bottom surface of the top-layer for the first rolling pass. The rolling direction is from left to right. Here the height reduction $(0.2 \%-0.6 \%)$ of the combined slab and the ratio between the thickness of the layers (the thick-top layer is twice as thick as the thin one) are varied in accordance with the industrial processing. For a thin top-layer (left subfigure in Figure 4) or a great height reduction the normal stresses and the surface enlargement in the contact zone of the layers are big which leads to a better bonding development at the access and center of the roll gap. This scenario is also visible at points D and C in Figure 2. Unfortunately, this process parameter setting has negative effect on the delivery side of roll gap. In this case an unfavorable combination of compression/tensile stresses and shear stresses, also visible in Figure 2 around point B, can lead to failure of an initially bonded contact point. Therefore only small height reductions of $0.2 \%$ and $0.3 \%$ lead to acceptable bonding behavior for the thin top-layer whereas for $0.4 \%, 0.5 \%$ and $0.6 \%$ height reduction no bonding is attainable. 


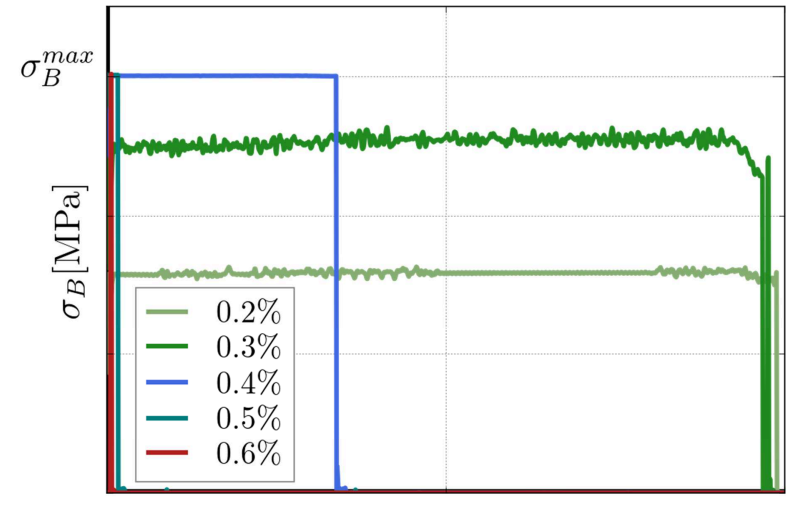

Thin Top-layer: bottom surface length

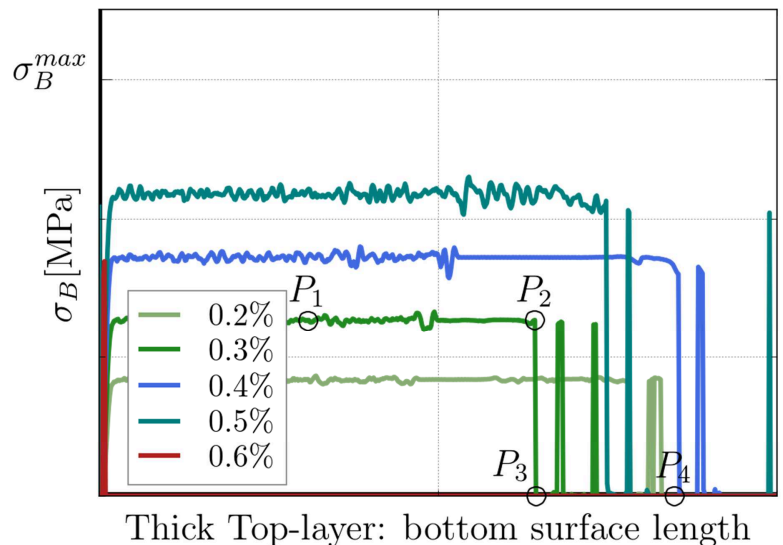

Thick Top-layer: bottom surface length

Figure 4: Snapshot of the reached bond strength $\sigma_{\mathrm{B}}$ and bonded length after rolling along the bottom surface of the top-layer for different height reductions and two top layer thicknesses.

The bonding/de-bonding effect within the roll gap can be observed especially well for the thick top-layer (right subfigure in Figure 4) at the right-hand side near the slab head. As visible in the distinct peaks of the curves, forming loads lead to bonding at the access and center of the roll gap but can lead to immediate failure on the delivery side of the roll gap. As the rolling operation reaches a steady state the fluctuations cease. Considering the nonlinear effects in the model (e.g. the material stress-strain responses) the difference in the bonded length for different height reductions is not surprising. Big height reductions induce high bonding stresses but also can initiate failure due to significant tension and shear loads on roll gap exit. Low height reductions on the other hand have little failure tendency but a general low bond strength, which also is vulnerable for failure. Thus, finding the best solution must be part of a numerical optimization process. Particularly the curves with thick top-layer and height reductions of $0.2 \%, 0.3 \%$ and $0.4 \%$ show an unsteady evolution of the bonded length of the two slabs, caused by the contradicting effects described above.
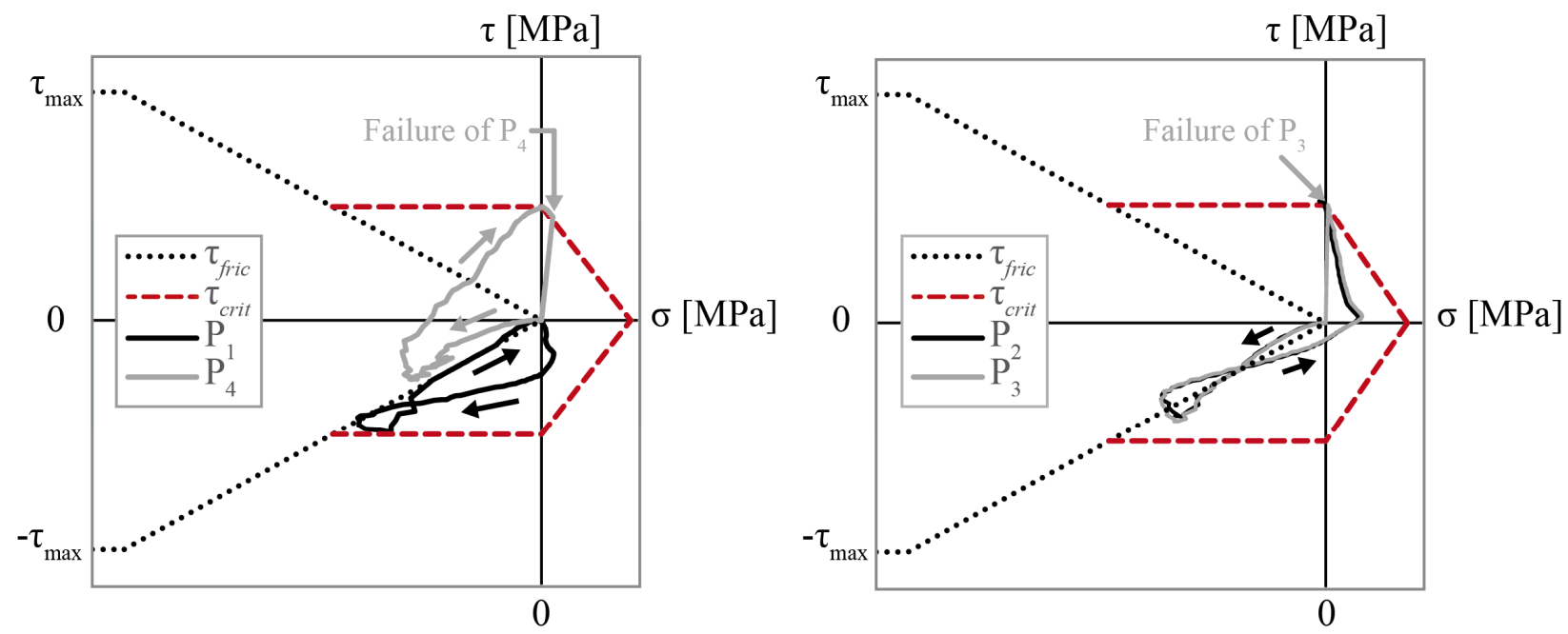

Figure 5: Evolution of the stress state within the normal-tangential stress space for the points $\mathrm{P}_{1}-\mathrm{P}_{4}$. $\mathrm{P}_{1}$ and $\mathrm{P}_{4}$ are distinct. $\mathrm{P}_{1}$ lies in a "safe" region and $\mathrm{P}_{4}$ fails clearly. Also $\mathrm{P}_{2}$ and $\mathrm{P}_{3}$ show narrow difference due to their direct neighbourship, only $\mathrm{P}_{3}$ fails.

For a better understanding of the local evolution in the roll gap Figure 5 shows typical trajectories within the normal-tangential stress space for the four interface nodes $\mathrm{P}_{1}-\mathrm{P}_{4}$ marked in the right subfigure of Figure 4. Again the red curves represent the critical border separating bonded and de-bonded states. Right after the entrance into the roll gap compressive and shear stresses dominate, accordingly bond strength evolves and the point trajectory is to the left and down. After passing the neutral point towards the delivery side the pressure drops and tensile stresses may occur. 
Hence, the point trajectory is directed towards the right and up. In Figure 5 two pairs of points are shown. The points $\mathrm{P}_{1}$ and $\mathrm{P}_{4}$ are distinct whereas $\mathrm{P}_{2}$ and $\mathrm{P}_{3}$ are in direct neightbourship. Accordingly the difference in slope of $\mathrm{P}_{2}$ and $\mathrm{P}_{3}$ is much smaller than the difference of $\mathrm{P}_{1}$ and $\mathrm{P}_{4}$. Also the points $\mathrm{P}_{3}$ and $\mathrm{P}_{4}$ show distinct slops, they fail in a comparable tensile/shear stress regime.

\section{Conclusion and Outlook}

In the current paper a FE-model to describe bond formation and failure was introduced based on the Abaqus user subroutine UINTER. Using this routine it is possible to analyze the bond formation in the first passes of a hot roll bonding process of aluminum alloys. The model shows that finding the height reduction best suitable for bonding in the first pass is not trivial due to the complex nature of the underlying processes. On the one hand bond strength can be increased with more height reduction due to greater surface enlargement, on the other hand greater height reduction increases the tensile and shear stresses and at the delivery side of the roll gap these combined stresses can break an already established bond. A level of process understanding that includes the evolution in the roll gap would not be possible with common elementary models.

For the future it is important to also incorporate the thermal interaction between the two layers instead of assuming isothermal conditions as temperature has a large impact in latter passes. Additionally the FE-model needs to be adopted for multi-pass rolling schedules to achieve the long term goal of predictive roll schedule design using simulations.

\section{Acknowledgements}

The authors would like to thank the Deutsche Forschungsgemeinschaft (DFG) for funding of the project "Walzplattieren von Metallen mit stark unterschiedlicher Festigkeit - Analyse der Verbindungsentstehung und modellbasierte Entwicklung neuer Prozessprinzipien" received within the priority program SPP1640. The authors are also grateful to Hydro R\&D, Bonn, for the scientific exchange and the fruitful discussions regarding roll bonding.

\section{References}

[1] Suzuki, H., Araki, J., Aiba, M., Shintani, K., 1974. An analytical study on mechanics in roll bonding of double-layer metal sheets. J. JSTP 15, 931-937.

[2] Lee, S.-H., Lee, D.N., 1991. Slab analysis of roll bonding of silver clad phosphor bronze sheets. Mater. Sci. Technol. 7, 1042-1050.

[3] Avitzur, B., Pachla, W., 1986. The upper bound approach to plain strain problems using linear and rotational velocity fields. Part 1. Basic concepts. J. Eng. Ind.Trans. ASME, 295-306.

[4] Manesh, H.D., Taheri, A.K., 2005. Theoretical and experimental investigation of cold rolling of tri-layer strip. J. Mater. Process. Technol. 166, 163-172.

[5] Schmidtchen, M., 2004. Walzplattieren von flächigen Mehrlagenverbunden - Experiment und Theorie in: Kawalla, R. (Hrsg.): MEFORM 2004: Technologie der Werkstoffverbundherstellung durch Umformen, Freiberg, 29-52

[6] Bay, N., Bjerregaard, H., Petersen, S., dos Santos, C., 1994. Cross shear roll bonding. J.Mater. Process. Technol. 45, 1-6.

[7] Bambach, M., Pietryga, M., Mikloweit, A., Hirt, G., 2014. A finite element framework for the evolution of bond strength in joining-by-forming processes, Journal of Materials Processing Technology 214, 2156-2168.

[8] Mikloweit, A., Bambach, M., Pietryga, M., Hirt, G., 2014. Development of a Testing Procedure to Determine the Bond Strength in Joining-by-Forming Processes, AMR 966-967, 481-488. 